\title{
1.92 Angstrom Zinc-Free APOBEC3F Catalytic Domain Crystal Structure
}

Nadine M. Shaban, ${ }^{1, \#^{*}}$ Ke Shi, ${ }^{1 \#}$ Ming Li, ${ }^{1}$ Hideki Aihara, ${ }^{1}$ and Reuben S. Harris ${ }^{1,2,{ }^{*}}$

${ }^{1}$ Department of Biochemistry, Molecular Biology, and Biophysics, Masonic Cancer

Center, Institute for Molecular Virology, University of Minnesota, Minneapolis, MN

55455

${ }^{2}$ Howard Hughes Medical Institute, University of Minnesota, Minneapolis, MN 55455

${ }^{\#}$ Equal Contributions

*Correspondence: nmshaban@umn.edu; rsh@umn.edu 


\begin{abstract}
The APOBEC3 family of DNA cytosine deaminases is capable of restricting the replication of HIV-1 and other pathogens. Here we report a 1.92 angstrom resolution crystal structure of the Vif-binding and catalytic domain of APOBEC3F (A3F). This structure is distinct from previously published APOBEC and phylogentically related deaminase structures, as it is the first without zinc in the active site. We determined an additional structure containing zinc in the same crystal form that allows direct comparison with the zinc-free structure. In the absence of zinc, the conserved active site residues that normally participate in zinc coordination show unique conformations, including a 90 degree rotation of His249 and disulfide bond formation between Cys280 and Cys283. We found that zinc coordination is influenced by $\mathrm{pH}$ and treating the protein at low $\mathrm{pH}$ in crystallization buffer is sufficient to remove zinc. Zinc coordination and catalytic activity is reconstituted with the addition of zinc only in a reduced environment likely due to the two active site cysteines readily forming a disulfide bond when not coordinating zinc. We show that the enzyme is active in the presence of zinc and cobalt, but not with other divalent metals. These results unexpectedly demonstrate that zinc is not required for the structural integrity of $A 3 F$ and suggest that metal coordination may be a strategy for regulating the activity of A3F and related deaminases.
\end{abstract}

\title{
Abbreviations
}

A3, APOBEC3; A3F, APOBEC3F; A3Fctd9X, APOBEC3F c-terminal domain with 9 
mutations; TCEP, Tris (2-carboxyethyl) phosphine hydrochloride; PDB, Protein Data Bank.

\author{
Keywords \\ Cytosine deaminase; Zinc-free and zinc-bound APOBEC3 structures; HIV-1 restriction \\ factor; APOBEC3 regulation; X-Ray crystallography.
}

\title{
Introduction
}

Deamination of the nucleobase cytosine is an essential reaction in nucleotide metabolism, adaptive immunity through antibody diversification, and innate immunity to endogenous retroelements and exogenous retroviruses (reviewed in [1, 2]). A multitude of related enzymes catalyze this reaction including free base cytosine deaminases (CD), nucleoside cytidine deaminases (CDA), and polynucleotide RNA and singlestranded DNA cytosine deaminases (APOBEC1, AID, APOBEC3A-H), as well as RNA adenine deaminases (ADAR and ADAT) $[2,3]$. These enzymes share a common zinccoordinating motif but differ in surrounding structural elements that orchestrate each enzyme's specific biological function. Mutagenesis studies have demonstrated that conserved amino acids in the zinc-coordinating motif are essential for catalysis (reviews cited above). For instance, mutating the catalytic glutamate or the zinc-coordinating residues (three cysteines or, in some enzymes, two cysteines and one histidine) completely abolishes catalytic activity and, in most instances, also abrogates HIV-1 restriction activity (e.g., studies on A3F [4-6]). 
The mammalian APOBEC3 (A3) enzymes form a powerful arm of the overall innate immune defense that functions to protect against the spread of endogenous mobile elements and exogenous viruses $[1,7]$. The human A3 repertoire is comprised of 3 single domain and 4 double domain enzymes $[1,7]$. $A 3 A, A 3 C$, and $A 3 H$ are single domain $\mathrm{A} 3 \mathrm{~s}$, whereas $\mathrm{A} 3 \mathrm{~B}, \mathrm{~A} 3 \mathrm{D}, \mathrm{A} 3 \mathrm{~F}$, and $\mathrm{A} 3 \mathrm{G}$ are double domain $\mathrm{A} 3$ s consisting of a pseudocatalytic N-terminal domain (ntd) and a catalytic C-terminal domain (ctd). The ntd and ctd each have a single conserved zinc-coordinating motif and a similar overall 3-dimensional fold, but the former is thought to be inactive due to a smaller active site cavity [8]. The overall A3 domain fold consists of a central beta sheet flanked by 6 alpha helices including the 2 zinc-coordinating helices. The alpha 2 helix has a His-X-Glu motif and the alpha 3 helix has a Cys-Pro- $\mathrm{X}_{2-4}$-Cys motif that together coordinate a single zinc ion. This is best evidenced by crystal and solution structures of $A 3 A[9,10]$, A3Bctd [11], A3C [12], A3Fctd ([13-15] and this study), A3Gntd [16], and A3Gctd [1722]. Importantly, prior to the current work, all crystal structures have contained a single zinc ion coordinated by the His-X-Glu and Cys-Pro- $\mathrm{X}_{2-4}-$ Cys motifs.

Here, we use x-ray crystallography to pursue new structural insights for the ctd of the antiviral DNA cytosine deaminase A3F. Surprisingly, we were able to obtain a highresolution zinc-free structure, as well as a zinc-bound structure in the same space group. The zinc-free enzyme is inactive, as expected, but catalytic activity can be restored with the addition of zinc under reducing conditions. These data suggest that the activity of $\mathrm{A} 3 \mathrm{~F}$, and perhaps other deaminase family members, may be regulated through differential zinc-coordination. 


\section{Results \\ Optimizing A3Fctd Expression and Purification}

The A3s generally exhibit poor solubility in overexpression studies in E. coli, and therefore domain truncations and solubility-enhancing mutations have been utilized in most structural studies [13-22]. We previously determined the crystal structure of A3Fctd with 11 solubility-enhancing amino acid substitutions [13]. In this study, we reverted 2 of the substitutions (Ala314, Ala315) back to wild-type (Tyr314, GIn315) because these residues are in alpha helix 4 within the region implicated in Vif-binding $[12,23,24]$, and also near loop 7 which is important for local substrate selection [10, 19, 25-27]. The resulting GST-A3Fctd9x construct expressed well in E. coli BL21 (DE3) and purified to near homogeneity with binding to glutathione sepharose resin, treatment with HRV 3 C protease to remove A3Fctd9x from the GST-tag, and further purification with Mono-Q ion exchange chromatography (Methods).

\section{A3Fctd9x Crystallization and Structure Determination}

A3Fctd9x crystallized in two different conditions, one at $\mathrm{pH} 5.0$ and the other at pH 6.9 (Table 1, Methods). Representative crystals from each condition were screened for diffraction at the APS beamline (Chicago, IL) and solved with molecular replacement using the A3Fctd11x crystal structure (PDB 4IOU [13]) as a search model. Each condition yielded crystals that belonged to the same space group, $\mathrm{P} 2{ }_{1}$, with 2 molecules in the asymmetric unit. This is the first time an A3F structure has been obtained in this space group. One structure resolved at 1.92 angstroms and the other at 2.33 angstroms. The former is the highest resolution to-date for any A3F structure and any 
Z2-type A3 deaminase domain. As expected, each crystal structure was comprised of a conserved cytidine deaminase fold with a central 5-stranded beta-sheet flanked by 6 alpha-helices (Fig. 1a, 1c). The overall structures are similar to the 3 published A3Fctd crystal structures (PDB 4IOU, 4J4J, 3WUS; [13-15]) with the exception of the loop1 region, which adopts a short alpha-helix in our new structures (Fig. 1a, 1c). The functional relevance of this additional secondary structure is not known at this time. It is possible that this secondary structure is an artifact of crystal packing. Alternatively, this short element is clearly evident in both structures and, given the importance of loop 1 in engaging single-stranded DNA substrates as demonstrated by prior studies [17, 19, 25, 27-31], it may be relevant to the overall biological function of the enzyme.

Surprisingly, the new crystal structure from the low $\mathrm{pH}$ condition lacked zinc in the active site, whereas the other had a single zinc ion at the expected position (compare Fig. 1a \& b with 1c \& d). Despite this fundamental difference, the zinc-free and zinc-bound A3Fctd9x structures align with a root mean square deviation (rmsd) of $1.168 \AA$ (chains $A$, all atom rmsd generated by pymol), which demonstrates high structural integrity and concordance. Based on the ubiquity of zinc in all available structures and prior mutagenesis studies demonstrating the essential nature of the zinccoordinating residues, we had assumed that zinc was required for proper expression and protein folding. Clearly, this crystal structure demonstrates that the overall cytidine deaminase fold, at least for A3Fctd, can be maintained in the absence of zinc. To our knowledge this constitutes the first zinc-free deaminase structure not only for the A3 subfamily but also for the larger family of single base, nucleoside, nucleotide, and polynucleotide enzymes. 


\section{Active Site Differences Between Zinc-Free and Zinc-Bound A3Fctd Structures}

Structural comparisons of superimposed zinc-free and zinc-bound A3Fctd9x show major shifts in several active site amino acid residues and no significant changes anywhere else in the protein (Fig. 1b \& 1d, Fig. 2a). Mainly, in chain A of the zinc-free A3Fctd9x, the imidazole ring of His 249 is rotated by $\sim 90$ degrees and Cys280 and Cys283 are joined by a disulfide bond (Fig. 2a). In contrast, the active site residues in the zinc-bound A3Fctd9X structure adopt conformations similar to those observed for the active sites of zinc-containing structures for A3A, A3C, A3Gctd, and A3Gntd (Fig. 2b). The zinc ion is coordinated directly by Cys 280 and Cys283 located on alpha helix 3 and His249 located on alpha helix 2, and indirectly through a water molecule by Glu251 also located on alpha helix 2 (Fig. 1d). In the zinc-bound structure, the active site cysteine residues are most likely incapable of forming a disulfide bond because they are deprotonated in order to directly coordinate the zinc ion, and the histidine side chain is oriented to position the nitrogen of the imidazole ring to directly coordinate the zinc ion (Fig. 2a, 2b).

\section{Differences in Active Site Residues Between Chains in the Asymmetric Unit}

The asymmetric unit of both the zinc-bound and zinc-free A3Fctd9x consists of two molecules. Chain A and B interact mainly by a beta-sheet interaction analogous to the crossing of two swords (Fig. 3a). The crystallographic dimerization interface involves a cation-pie interaction formed by Trp233 on beta-2 of chain A and Arg235 on beta-2 of chain B (Fig. 3b). Interactions are also present between Arg235 on the beta-2 
of chain A and Glu193 of chain B (Fig. 3b). Overall, the two chains show high similarity with, again, the exception being differences in active site residues (Fig. 3a). In chain B of the zinc-free structure, there is no electron density for His249 suggesting high flexibility of this residue, and Cys280 and Cys283 are not within disulfide bonding distance as seen in chain A (Fig. 3c). A superposition of the active site residues of chain $A$ of the zinc-free structure, chain B of the zinc-free A3Fctd9x structure, and chain A of the zinc-bound structure show that the Cys280 and Cys283 in chain B of the zincfree A3Fctd9x structure adopts a configuration similar to the zinc-bound structure (Fig. 3c). Thus, the zinc-free A3Fctd9x crystal structure captured the active site in two different conformations; one in which the histidine is in an alternate conformation and the 2 cysteines form a disulfide bond (chain A), and another in which the histidine is highly flexible and the cysteines do not form a disulfide bond (chain B; Fig. 3c). It is possible that chain B active site conformation in the zinc-free A3Fctd9X structure precedes the conformation in chain A of the same structure. That is, the histidine becomes more ordered when the disulfide bond forms between active site cysteines. Both states highlight the inherent flexibility of the active site in the absence of zinc. This flexibility likely facilitates removal of zinc under the right conditions.

\section{Zinc Reconstitution Experiments}

We postulated that the low $\mathrm{pH}$ and/or the chemical composition of the crystallization condition caused the displacement of the zinc ion from A3Fctd9x. For example, a low $\mathrm{pH}$ environment may protonate the active site histidine and/or cysteines and trigger zinc removal. It is also possible that anions in the tacsimate crystallization 
condition (ammonium citrate tribasic, ammonium tartrate dibasic, DL-malic acid, malonic acid, sodium acetate trihydrate, sodium formate, and succinic acid) could have chelated zinc away from the protein. To test if a zinc-free enzyme could be reconstituted, we dialyzed purified A3Fctd9x into buffer containing $12 \%$ tacsimate at $\mathrm{pH} 5$ or $\mathrm{pH} 7$, and then both samples were dialyzed into a tacsimate-free buffer at $\mathrm{pH} 7.5$ for activity assays (see Methods). As a control, a portion of the same A3Fctd9x protein preparation was dialyzed in parallel into a tacsimate-free final buffer at $\mathrm{pH} 7.5$. The zinc composition of each A3Fctd9x preparation was then quantified. A significant reduction in zinc levels was only observed for the protein sample treated with tacsimate at $\mathrm{pH} 5$ (data not shown), indicating that low $\mathrm{pH}$ facilitates the removal of zinc.

We next assayed the catalytic activity of the zinc-free A3Fctd9x treated at pH 5 in the presence and absence of exogenous zinc and the reducing agent TCEP (this was chosen over alternatives such as DTT or $\beta$-mercaptoethanol to minimize the possibility of zinc chelation). We found that the untreated A3Fctd9x showed activity with and without zinc, further confirming that the purified protein prior to crystallization is bound to zinc (Fig. 4a). In contrast, A3Fctd9x treated with tacsimate at pH 5 was inactive without zinc and showed activity only with the addition of $1-10 \mu \mathrm{M}$ of zinc, and only in the presence of reducing agent (Fig. 4a). This further supports the inference that this protein was stripped of zinc by low $\mathrm{pH}$ treatment, and that the 2 cysteines likely formed a disulfide bond as seen in the crystal structure. Both the untreated A3Fctd9x and A3Fctd9x treated with tacsimate at $\mathrm{pH} 5$ lose activity at higher zinc concentrations, likely due to zinc-mediated precipitation of proteins [32, 33]. We further tested whether A3Fctd9x could utilize other divalent metals in place of zinc. Zinc-free A3Fctd9x (treated 
with tacsimate at $\mathrm{pH}$ 5) showed detectable activity with the addition of exogenous zinc or cobalt, but not with several other metals tested (Fig. 4b).

\section{Discussion}

Our new crystal structures of the catalytic and Vif-binding domain of A3F provide multiple insights into the overall structural biology and biochemistry of cytosine deaminases. The biggest surprise is that a high-resolution structure was obtained without zinc in the active site. All previous data of APOBEC crystal and NMR structures $[9,10,12-22,34,35]$, as well as structures of phylogenetically related enzymes (CD, CDD1, ADAR, ADAT [36-51]), indicate zinc coordination at the active site.. For all tested enzymes, mutagenesis studies have shown that the conserved zinc coordinating residues are essential for catalytic activity (e.g., A3F H249, E251, C280, and C283 [46]). However, the active site glutamic acid may be dispensable for zinc binding because changing this residue in $\mathrm{A} 3 \mathrm{~A}(\mathrm{E} 72 \mathrm{~A})$ or in the distantly related Blasticidin S. deaminase (E56Q) did not prevent the protein from coordinating zinc as evidenced by crystal structures $[9,52]$. Our structural and biochemical data indicate that zinc binding in the A3F catalytic domain is mainly influenced by ionic conditions and the redox state of the catalytic histidine and cysteine residues. Due to high levels amino acid homology and structural similarity, it is likely that other A3 family members are similarly susceptible to the surrounding environment.

Interestingly, our zinc-bound and zinc-free A3Fctd9X structures and existing A3Fctd structures all crystallized in different space groups [13-15]. Although it is tempting to infer biologically relevant interaction surfaces from crystal contacts, there 
are no common interaction mechanisms in or between molecules in the asymmetric unit among all structures, suggesting that these crystal interfaces are not biologically relevant dimerization or higher order oligomerization motifs. The most notable difference in crystal packing occurs between A3Fctd9X and A3Fctd11X. It was speculated that the beta1-beta2 region containing A3F11Xctd residues Lys226-His227-His228-Ser229, plays a role in autoregulation of catalytic activity as these residues from one molecule in the asymmetric unit are inserted into the active site of another near the catalytic zinc ion [13]. However, this interaction is absent in the A3Fctd9X structure suggesting that this may not be a conserved mode of regulation. However, we cannot rule out the possibility that these 4 residues participate in oligomerization or some other interaction, as these residues, and the beta-1/beta-2 region in general, do play a significant role in crystal packing interactions in all A3Fctd structures (although by different interaction mechanisms).

The prospect that the catalytic activity of A3F and related deaminases may be regulated through differential zinc coordination is intriguing. If local $\mathrm{pH}$ and/or redox state are linked to physiological responses, such as virus infections or inflammation, it could constitute a mechanism for activating deaminase activity at appropriate times or concentrating it in appropriate places, while leaving it inactive in the vast majority of cells most of the time. It is important to emphasize that differential zinc coordination could be achieved by changing the surrounding chemical environment and/or by a regulated protein-protein interaction. Such regulatory processes could help to minimize the contribution of APOBEC enzymes to undesirable processes such as somatic mutation in cancer. Indeed, recent studies have demonstrated that APOBEC 
mutagenesis is prevalent in many different tumor types, including breast, head/neck, lung, bladder, and cervical cancers (reviewed in [53-55]). The ntd of a key enzyme responsible, $\mathrm{APOBEC} 3 \mathrm{~B}$, has strong similarity with $\mathrm{A} 3 \mathrm{Fctd}$ and could be amendable to zinc regulation. However, it is unlikely that low $\mathrm{pH}$ alone is sufficient to remove the zinc in all A3s as evidenced by $\mathrm{A} 3 \mathrm{G}$, which is more active at $\mathrm{pH} 5.5$ [30]. Alternatively, A3s could be regulated through oxidation. Although the redox state of the A3 active site cysteines has yet to be investigated, our structure indicates that oxidation of cysteines and formation of a disulfide bond will prevent zinc coordination and block activity. Redox regulation of cysteine residues is observed in several zinc coordinating proteins, such as the inactivation of zinc fingers in transcription factors by oxidation of the cysteines that coordinate zinc (reviewed in [56]). Considering that the A3s maintain a proper fold in a zinc-free state in vitro, and loss of zinc-coordination and activity can be reversed, investigation into zinc-free A3s in vivo is warranted.

\section{Materials and Methods:}

\section{Protein Expression}

A3Fctd11X 185-373 cloned into pGEX6P-2 was generated as previously described [13]. Site directed mutagenesis was used to revert residue Ala-314 to Tyr, and Ala-315 to Gln. E. coli BL21 (DE3) were used for overexpression. Cells transformed with expression plasmid were grown in LB medium with $100 \mu \mathrm{g} / \mathrm{ml}$ of ampicillin. Cells were grown to OD $\sim 0.8$, cooled to $16^{\circ} \mathrm{C}$ and induced with $1 \mathrm{mM}$ IPTG. Cells were harvested $\sim 16 \mathrm{hrs}$ later and resuspended in $50 \mathrm{mM}$ Tris $\mathrm{pH} 7.5,300 \mathrm{mM} \mathrm{NaCl}$, and $5 \mathrm{mM}$ DTT, $100 \mu \mathrm{g} / \mathrm{ml}$ of lysozyme and disrupted with sonication. Cells were clarified at $14,000 \mathrm{xg}$ 
for 20min., and supernatant was collected and incubated with glutathione resin (GE Healthcare). GST-A3F bound resin was washed with 50mM Tris pH 7.5, 100mM NaCl, and $5 \mathrm{mM}$ DTT. Resin was incubated with HRV $3 \mathrm{C}$ protease overnight at $4^{\circ} \mathrm{C}$ for oncolumn cleavage of A3Fctd9x. Cleaved A3Fctd9x was further purified with a GEHealthcare HiTrap Q HP $5 \mathrm{~mL}$ column and eluted with a stepwise gradient of sodium chloride. A3Fctd9x was concentrated to $\sim 10 \mathrm{mg} / \mathrm{ml}$ in final buffer of $20 \mathrm{mM}$ Tris- $\mathrm{HCl} \mathrm{pH}$ 7.5, $300 \mathrm{mM} \mathrm{NaCl}$ and 5mM DTT.

\section{Protein Crystallization}

Protein crystals were grown by sitting drop vapor diffusion method in 96 well crystal trays. Approximately $10 \mathrm{mg} / \mathrm{ml}$ of A3Fctd9X was mixed with equal volumes of crystallization buffer. Protein crystals of the zinc-free A3Fctd9X grew in $12 \%(\mathrm{v} / \mathrm{v})$ tacsimate $\mathrm{pH} 5$ (Hampton Research), 10\% PEG 3350, and $10 \%$ glycerol at $18^{\circ} \mathrm{C}$. Protein crystals of the zinc-bound A3Fctd9X structure were grown in $200 \mathrm{mM}$ magnesium chloride and 20\% PEG 3350 at $18^{\circ} \mathrm{C}$. Crystals were cryo-protected with $20 \%$ glycerol. Crystals grew within a week.

\section{Data Collection and Structure Determination}

Crystals were screened and data were collected at 24-ID-C and 24-ID-E beamlines at the Northeastern Collaborative Access Team (NE-CAT) at the Advanced Photon Source in Argonne National Laboratory. Data was processed with HKL2000 Suite [57]. Molecular replacement in PHASER [58] was performed using A3Fctd11x crystal structure (PDB 4IOU [13]) as a search model. The model was built in Coot [59] and refined with PHENIX [60]. The Zn-free and Znbound structures were refined identically with the exception that, for the final step of the $\mathrm{Zn}$-free 
structure refinement was done with riding $\mathrm{H}$-atoms which caused the $\mathrm{R}$-work and $\mathrm{R}$-free values to decrease $(0.71 \%$ and $0.30 \%$, respectively). Therefore, the $\mathrm{H}$-atoms were kept in the final model. However, for the $\mathrm{Zn}$-bound structure, refinement with riding $\mathrm{H}$-atoms increased the $\mathrm{R}$ work and R-free slightly, and therefore this structure was refined without $\mathrm{H}$-atoms. Data collection and refinement statistics are shown in Table 1.

\title{
Dialysis of A3Fctd9x in Tacsimate pH 5 and 7
}

A3Fctd9x was purified as described above. Equal volumes of the protein was dialyzed into $500 \mathrm{mls}$ of buffer containing $300 \mathrm{mM} \mathrm{NaCl}, 10 \%$ glycerol and $12 \%(\mathrm{v} / \mathrm{v})$ tacsimate (Hampton Research) at $\mathrm{pH} 5$ or $\mathrm{pH} 7$, at $4^{\circ} \mathrm{C}$ for $\sim 48$ hrs. Slight precipitation was observed in the $\mathrm{pH} 5$ sample. The two protein samples, and A3Fctd9x (purified protein not treated with tacsimate) were then dialyzed into $1000 \mathrm{mls}$ of a tacsimate-free buffer containing $300 \mathrm{mM} \mathrm{NaCl}, 10 \%$ glycerol, and $50 \mathrm{mM}$ Tris- $\mathrm{HCl} \mathrm{pH} 7.5$, at $4^{\circ} \mathrm{C}$ for $\sim 48 \mathrm{hrs}$. The dialysis buffer was exchanged once. After dialysis the samples were centrifuged to remove any precipitated protein, and protein concentration was measured. Although slight protein precipitation was observed in the low $\mathrm{pH}$ sample, no significant protein loss was detected. Zinc content was assessed using zinc colorimetric quantification kit (Abcam).

\begin{abstract}
A3Fctd9x Deaminase Activity Assay
A3Fctd9x activity was tested using a DNA oligo-cleavage deamination assay as described in [61]. $10 \mu \mathrm{M}$ of each A3Fctd9X sample was mixed with 200nM of single stranded fluorescently labeled (6-FAM) oligo substrate: 5'-
\end{abstract} ATTATTATTATTCAAATGGATTTATTTATTTATTTATTTATTT-FAM-3', and 0.02 units of UDG 
(NEB), in reaction buffer containing $50 \mathrm{mM}$ Tris $\mathrm{pH} 7.4,100 \mathrm{mM} \mathrm{NaCl}, 0.005 \%$ Triton $\mathrm{X}$ 100 (reducing agent and metal ion was added to each reaction as indicated in Figure 4). The reaction mixture was incubate for $2 \mathrm{hrs}$, and then treated with a final concentration of $100 \mathrm{mM} \mathrm{NaOH}$ for $5 \mathrm{~min}$. at $95^{\circ} \mathrm{C}$ to cleave the abasic site generated by UDG excision of the uracil base. $2 X$ denaturing PAGE loading dye was added to each reaction, and samples were run on $20 \%$ TBE-urea polyacrylamide gels to separate the product and substrate. Gels were imaged using a Fuji FLA-5000 scanner.

\author{
Accession codes \\ The structure factors and atomic coordinates for the zinc-free and zinc-bound A3Fctd9X \\ structures have been deposited in the Protein Data Bank with PDB ID 5HX4 and 5HX5, \\ and will be released upon publication.
}

\title{
Author Contributions
}

NMS and RSH designed experiments and wrote the paper. NMS purified and crystallized the protein. KS collected and processed the diffraction data. KS and NMS determined the structures. ML performed the oligo-cleavage activity assay. All authors analyzed data, edited the paper, and approved the final version.

\section{Acknowledgments}

The Harris lab is supported by NIH NIAID AI064046, NIGMS GM091743, and HHMI. The Aihara lab is supported by NIH NIGMS grants GM095558 and GM109770. 


\section{Conflict of Interest}

$\mathrm{RSH}$ is a cofounder of ApoGen Biotechnologies Inc. The other authors declare that they

have no conflicts of interest with the contents of this article.

\section{References}

[1] EW Refsland, RS Harris. The APOBEC3 family of retroelement restriction factors. Current topics in microbiology and immunology. 2013;371:1-27.

[2] SG Conticello. The AID/APOBEC family of nucleic acid mutators. Genome biology. 2008;9:229.

[3] RA Goodman, MR Macbeth, PA Beal. ADAR proteins: structure and catalytic mechanism. Current topics in microbiology and immunology. 2012;353:1-33.

[4] JS Albin, WL Brown, RS Harris. Catalytic activity of APOBEC3F is required for efficient restriction of Vif-deficient human immunodeficiency virus. Virology. 2014;450-451:49-54.

[5] RK Holmes, FA Koning, KN Bishop, MH Malim. APOBEC3F can inhibit the accumulation of HIV-1 reverse transcription products in the absence of hypermutation. Comparisons with APOBEC3G. The Journal of biological chemistry. 2007;282:2587-95.

[6] E Miyagi, CR Brown, S Opi, M Khan, R Goila-Gaur, S Kao, et al. Stably expressed APOBEC3F has negligible antiviral activity. Journal of virology. 2010;84:11067-75.

[7] RS Harris, JP Dudley. APOBECs and virus restriction. Virology. 2015;479-480:13145.

[8] SM Shandilya, MF Bohn, CA Schiffer. A computational analysis of the structural determinants of APOBEC3's catalytic activity and vulnerability to HIV-1 Vif. Virology. 2014;471-473:105-16.

[9] MF Bohn, SM Shandilya, TV Silvas, EA Nalivaika, T Kouno, BA Kelch, et al. The ssDNA Mutator APOBEC3A Is Regulated by Cooperative Dimerization. Structure. 2015;23:903-11.

[10] M Mitra, K Hercik, IJ Byeon, J Ahn, S Hill, K Hinchee-Rodriguez, et al. Structural determinants of human APOBEC3A enzymatic and nucleic acid binding properties. Nucleic acids research. 2014;42:1095-110.

[11] K Shi, MA Carpenter, K Kurahashi, RS Harris, H Aihara. Crystal Structure of the DNA Deaminase APOBEC3B Catalytic Domain. The Journal of biological chemistry. 2015;290:28120-30.

[12] S Kitamura, H Ode, M Nakashima, M Imahashi, Y Naganawa, T Kurosawa, et al. The APOBEC $3 \mathrm{C}$ crystal structure and the interface for HIV-1 Vif binding. Nature structural \& molecular biology. 2012;19:1005-10.

[13] MF Bohn, SM Shandilya, JS Albin, T Kouno, BD Anderson, RM McDougle, et al. Crystal structure of the DNA cytosine deaminase APOBEC3F: the catalytically active and HIV-1 Vif-binding domain. Structure. 2013;21:1042-50.

[14] KK Siu, A Sultana, FC Azimi, JE Lee. Structural determinants of HIV-1 Vif susceptibility and DNA binding in APOBEC3F. Nature communications. 2013;4:2593. 
[15] M Nakashima, H Ode, T Kawamura, S Kitamura, Y Naganawa, H Awazu, et al. Structural Insights into HIV-1 Vif-APOBEC3F Interaction. Journal of virology. 2015;90:1034-47.

[16] T Kouno, EM Luengas, M Shigematsu, SM Shandilya, J Zhang, L Chen, et al. Structure of the Vif-binding domain of the antiviral enzyme APOBEC3G. Nature structural \& molecular biology. 2015;22:485-91.

[17] KM Chen, E Harjes, PJ Gross, A Fahmy, Y Lu, K Shindo, et al. Structure of the DNA deaminase domain of the HIV-1 restriction factor APOBEC3G. Nature. 2008;452:116-9.

[18] SM Shandilya, MN Nalam, EA Nalivaika, PJ Gross, JC Valesano, K Shindo, et al. Crystal structure of the APOBEC3G catalytic domain reveals potential oligomerization interfaces. Structure. 2010;18:28-38.

[19] LG Holden, C Prochnow, YP Chang, R Bransteitter, L Chelico, U Sen, et al. Crystal structure of the anti-viral APOBEC3G catalytic domain and functional implications. Nature. 2008;456:121-4.

[20] A Furukawa, T Nagata, A Matsugami, Y Habu, R Sugiyama, F Hayashi, et al. Structure, interaction and real-time monitoring of the enzymatic reaction of wild-type APOBEC3G. The EMBO journal. 2009;28:440-51.

[21] M Li, SM Shandilya, MA Carpenter, A Rathore, WL Brown, AL Perkins, et al. Firstin-class small molecule inhibitors of the single-strand DNA cytosine deaminase APOBEC3G. ACS chemical biology. 2012;7:506-17.

[22] X Lu, T Zhang, Z Xu, S Liu, B Zhao, W Lan, et al. Crystal structure of DNA cytidine deaminase $A B O B E C 3 G$ catalytic deamination domain suggests a binding mode of full-length enzyme to single-stranded DNA. The Journal of biological chemistry. 2015;290:4010-21.

[23] AM Land, NM Shaban, L Evans, JF Hultquist, JS Albin, RS Harris. APOBEC3F determinants of HIV-1 Vif sensitivity. Journal of virology. 2014;88:12923-7.

[24] JS Albin, RS LaRue, JA Weaver, WL Brown, K Shindo, E Harjes, et al. A single amino acid in human APOBEC3F alters susceptibility to HIV-1 Vif. The Journal of biological chemistry. 2010;285:40785-92.

[25] A Rathore, MA Carpenter, O Demir, T Ikeda, M Li, NM Shaban, et al. The local dinucleotide preference of APOBEC3G can be altered from 5'-CC to 5'-TC by a single amino acid substitution. Journal of molecular biology. 2013;425:4442-54.

[26] MA Carpenter, E Rajagurubandara, P Wijesinghe, AS Bhagwat. Determinants of sequence-specificity within human AID and APOBEC3G. DNA repair. 2010;9:57987.

[27] RM Kohli, SR Abrams, KS Gajula, RW Maul, PJ Gearhart, JT Stivers. A portable hot spot recognition loop transfers sequence preferences from APOBEC family members to activation-induced cytidine deaminase. The Journal of biological chemistry. 2009;284:22898-904.

[28] V Caval, MS Bouzidi, R Suspene, H Laude, MC Dumargne, A Bashamboo, et al. Molecular basis of the attenuated phenotype of human APOBEC3B DNA mutator enzyme. Nucleic acids research. 2015;43:9340-9.

[29] Y Bulliard, I Narvaiza, A Bertero, S Peddi, UF Rohrig, M Ortiz, et al. Structurefunction analyses point to a polynucleotide-accommodating groove essential for APOBEC3A restriction activities. Journal of virology. 2011;85:1765-76. 
[30] S Harjes, WC Solomon, M Li, KM Chen, E Harjes, RS Harris, et al. Impact of H216 on the DNA binding and catalytic activities of the HIV restriction factor APOBEC3G. Journal of virology. 2013;87:7008-14.

[31] Y Fu, F Ito, G Zhang, B Fernandez, H Yang, XS Chen. DNA cytosine and methylcytosine deamination by APOBEC3B: enhancing methylcytosine deamination by engineering APOBEC3B. The Biochemical journal. 2015;471:25-35.

[32] TH Yang, JL Cleland, X Lam, JD Meyer, LS Jones, TW Randolph, et al. Effect of zinc binding and precipitation on structures of recombinant human growth hormone and nerve growth factor. Journal of pharmaceutical sciences. 2000;89:1480-5.

[33] RE Lovrien, D Matulis. Selective precipitation of proteins. Current protocols in protein science / editorial board, John E. Coligan ... [et al.]. 2001; Chapter 4:Unit 45.

[34] IJ Byeon, J Ahn, M Mitra, CH Byeon, K Hercik, J Hritz, et al. NMR structure of human restriction factor APOBEC3A reveals substrate binding and enzyme specificity. Nature communications. 2013;4:1890.

[35] C Prochnow, R Bransteitter, MG Klein, MF Goodman, XS Chen. The APOBEC-2 crystal structure and functional implications for the deaminase AID. Nature. 2007;445:447-51.

[36] L Betts, S Xiang, SA Short, R Wolfenden, CW Carter, Jr. Cytidine deaminase. The 2.3 A crystal structure of an enzyme: transition-state analog complex. Journal of molecular biology. 1994;235:635-56.

[37] SJ Chung, JC Fromme, GL Verdine. Structure of human cytidine deaminase bound to a potent inhibitor. Journal of medicinal chemistry. 2005;48:658-60.

[38] Y Elias, RH Huang. Biochemical and structural studies of A-to-I editing by tRNA:A34 deaminases at the wobble position of transfer RNA. Biochemistry. 2005;44:12057-65.

[39] AH Teh, M Kimura, M Yamamoto, N Tanaka, I Yamaguchi, T Kumasaka. The 1.48 A resolution crystal structure of the homotetrameric cytidine deaminase from mouse. Biochemistry. 2006;45:7825-33.

[40] K Xie, MP Sowden, GS Dance, AT Torelli, HC Smith, JE Wedekind. The structure of a yeast RNA-editing deaminase provides insight into the fold and function of activation-induced deaminase and APOBEC-1. Proceedings of the National Academy of Sciences of the United States of America. 2004;101:8114-9.

[41] E Johansson, N Mejlhede, J Neuhard, S Larsen. Crystal structure of the tetrameric cytidine deaminase from Bacillus subtilis at $2.0 \mathrm{~A}$ resolution. Biochemistry. 2002;41:2563-70.

[42] TP Ko, JJ Lin, CY Hu, YH Hsu, AH Wang, SH Liaw. Crystal structure of yeast cytosine deaminase. Insights into enzyme mechanism and evolution. The Journal of biological chemistry. 2003;278:19111-7.

[43] HC Losey, AJ Ruthenburg, GL Verdine. Crystal structure of Staphylococcus aureus tRNA adenosine deaminase TadA in complex with RNA. Nature structural \& molecular biology. 2006;13:153-9.

[44] MR Macbeth, HL Schubert, AP Vandemark, AT Lingam, CP Hill, BL Bass. Inositol hexakisphosphate is bound in the ADAR2 core and required for RNA editing. Science. 2005;309:1534-9. 
[45] DK Wilson, FB Rudolph, FA Quiocho. Atomic structure of adenosine deaminase complexed with a transition-state analog: understanding catalysis and immunodeficiency mutations. Science. 1991;252:1278-84.

[46] S Xiang, SA Short, R Wolfenden, CW Carter, Jr. The structure of the cytidine deaminase-product complex provides evidence for efficient proton transfer and ground-state destabilization. Biochemistry. 1997;36:4768-74.

[47] M Kuratani, R Ishii, Y Bessho, R Fukunaga, T Sengoku, M Shirouzu, et al. Crystal structure of tRNA adenosine deaminase (TadA) from Aquifex aeolicus. The Journal of biological chemistry. 2005;280:16002-8.

[48] S Xiang, SA Short, R Wolfenden, CW Carter, Jr. Transition-state selectivity for a single hydroxyl group during catalysis by cytidine deaminase. Biochemistry. 1995;34:4516-23.

[49] E Johansson, J Neuhard, M Willemoes, S Larsen. Structural, kinetic, and mutational studies of the zinc ion environment in tetrameric cytidine deaminase. Biochemistry. 2004;43:6020-9.

[50] GC Ireton, G McDermott, ME Black, BL Stoddard. The structure of Escherichia coli cytosine deaminase. Journal of molecular biology. 2002;315:687-97.

[51] J Kim, V Malashkevich, S Roday, M Lisbin, VL Schramm, SC Almo. Structural and kinetic characterization of Escherichia coli TadA, the wobble-specific tRNA deaminase. Biochemistry. 2006;45:6407-16.

[52] T Kumasaka, M Yamamoto, M Furuichi, M Nakasako, AH Teh, M Kimura, et al. Crystal structures of blasticidin $S$ deaminase (BSD): implications for dynamic properties of catalytic zinc. The Journal of biological chemistry. 2007;282:37103-11.

[53] T Helleday, S Eshtad, S Nik-Zainal. Mechanisms underlying mutational signatures in human cancers. Nature reviews. Genetics. 2014;15:585-98.

[54] C Swanton, N McGranahan, GJ Starrett, RS Harris. APOBEC Enzymes: Mutagenic Fuel for Cancer Evolution and Heterogeneity. Cancer discovery. 2015;5:704-12.

[55] SA Roberts, DA Gordenin. Hypermutation in human cancer genomes: footprints and mechanisms. Nature reviews. Cancer. 2014;14:786-800.

[56] NM Giles, AB Watts, GI Giles, FH Fry, JA Littlechild, C Jacob. Metal and redox modulation of cysteine protein function. Chemistry \& biology. 2003;10:677-93.

[57] Z Otwinowski, W Minor. Processing of X-ray diffraction data collected in oscillation mode. Methods in Enzymology, Macromolecular Crystallography, part A. p. 307-326, C.W. Carter, Jr. \& R.M. Sweets, Eds. New York: Academic Press; 1997.

[58] AJ McCoy, RW Grosse-Kunstleve, PD Adams, MD Winn, LC Storoni, RJ Read. Phaser crystallographic software. Journal of applied crystallography. 2007;40:65874.

[59] P Emsley, B Lohkamp, WG Scott, K Cowtan. Features and development of Coot. Acta crystallographica. Section D, Biological crystallography. 2010;66:486-501.

[60] PD Adams, PV Afonine, G Bunkoczi, VB Chen, IW Davis, N Echols, et al. PHENIX: a comprehensive Python-based system for macromolecular structure solution. Acta crystallographica. Section D, Biological crystallography. 2010;66:213-21.

[61] MA Carpenter, M Li, A Rathore, L Lackey, EK Law, AM Land, et al. Methylcytosine and normal cytosine deamination by the foreign DNA restriction enzyme APOBEC3A. The Journal of biological chemistry. 2012;287:34801-8. 


\section{Figure Legends}

Fig. 1.

Crystal structures of zinc-free and zinc-bound A3Fctd9x. (a) Zinc-free A3Fctd9x adopts a cytidine deaminase fold. (b) A simulated annealing 2Fo-Fc composite omit electron density map (blue mesh) contoured at 1 sigma showing no density for zinc in the active site of the zinc-free A3Fctd9x structure. (c) Zinc-bound A3Fctd9x adopts a cytidine deaminase fold. A zinc ion is coordinated by active site residues His249, Glu251, Cys280, and Cys283. (d) A simulated annealing 2Fo-Fc composite omit electron density map (blue mesh) contoured at 1 sigma, and anomalous difference fourier map contoured at 5 sigma (orange mesh), showing clear density for zinc (gray sphere) in the active site of the zinc-bound A3Fctd9x structure. The water molecule is represented by a red sphere.

\section{Fig. 2.}

Active site comparison of zinc-free and zinc-bound A3 structures. (a) Superposition of zinc-free (orange) and zinc-bound A3Fctd9x (grey) crystal structures. Active site closeup shows positioning of catalytic His249, and disulfide bond formation between Cys280 and Cys283 in zinc-free A3Fctd9X. (b) Structural comparison of zinc-free and zincbound A3Fctd9x (orange, grey) active sites with A3C (cyan, PDB 3VOW), A3Gctd (green, PDB 3IR2) active site residues. 


\section{Fig. 3.}

Crystal packing of A3Fctd9x structures. (a) Zinc-free (represented) and zinc-bound A3Fctd9x pack as two molecules in the asymmetric unit. (b) Main interactions between the two chains in the asymmetric unit are by the beta-sheet of the cytidine deaminase fold. (c) Superposition of the active site residues of the zinc-free A3Fctd9x chain A (orange), zinc-free A3Fctd9x chain B (yellow), and zinc-bound A3Fctd9x chain A (grey). In chain B of the zinc-free A3Fctd9x structure, His249 is not visible, and Cys280 and Cys283 do not form a disulfide bond.

Fig. 4.

A3Fctd9x deaminase activity assay. (a) Oligo-cleavage deaminase assay in presence and absence of $\mathrm{ZnCl}_{2}$ with purified $\mathrm{A} 3 \mathrm{Fctd} 9 \mathrm{X}$ (control), and $\mathrm{A} 3 \mathrm{Fctd} 9 \mathrm{x}$ treated with tacsimate $\mathrm{pH} 5$, with and without reducing agent (TCEP). (b) Oligo cleavage deaminase assay with metal ions. Deaminase activity was tested for A3Fctd9X not treated with tacsimate (control), and for A3Fctd9X treated with tacsimate $\mathrm{pH} 5$ and $\mathrm{pH} 7$. 
Table 1. Data collection and refinement statistics.

A3Fctd9x Zinc-free

\section{Diffraction data}

\begin{tabular}{|c|c|c|}
\hline Wavelength $(\AA)$ & 0.979 & 0.979 \\
\hline Resolution range $(\AA)$ & $49.91-1.92(2.00-1.92)$ & $45.40-2.33(2.42-2.33)$ \\
\hline Space group & $\mathrm{P} 2_{1}$ & $\mathrm{P} 2_{1}$ \\
\hline \multicolumn{3}{|l|}{ Unit cell } \\
\hline$a, b, c(\AA)$ & $36.12,51.29,99.81$ & $35.93,50.84,100.8$ \\
\hline$\alpha, \beta, \gamma\left({ }^{\circ}\right)$ & $90,90.85,90$ & $90,90.74,90$ \\
\hline Total reflections & $82386(4247)$ & $47116(4943)$ \\
\hline Unique reflections & $26142(1828)$ & $15324(1559)$ \\
\hline Multiplicity & $3.2(2.3)$ & $3.1(3.2)$ \\
\hline Completeness (\%) & $93.1(62.8)$ & $96.8(99.0)$ \\
\hline Mean $/ / \sigma_{1}$ & $12.0(0.80)$ & $6.10(1.04)$ \\
\hline$R_{\text {merge }}$ & $0.054(0.91)$ & $0.12(1.12)$ \\
\hline $\mathrm{CC}_{1 / 2}$ & $0.999(0.340)$ & $0.981(0.433)$ \\
\hline \multicolumn{3}{|l|}{ Refinement } \\
\hline Reflections & $25899(1826)$ & $15303(1559)$ \\
\hline $\mathrm{R}_{\text {work }}(\%)$ & $18.48(31.37)$ & $22.68(31.48)$ \\
\hline $\mathrm{R}_{\text {free }}(\%)$ & $21.37(31.69)$ & $26.44(35.37)$ \\
\hline Number of atoms & 3035 & 2951 \\
\hline Macromolecules & 2918 & 2888 \\
\hline Protein residues & 345 & 342 \\
\hline \multicolumn{3}{|l|}{ r.m.s.d. } \\
\hline Bonds length $(\AA)$ & 0.011 & 0.003 \\
\hline Bonds angle $\left({ }^{\circ}\right)$ & 0.96 & 0.60 \\
\hline \multicolumn{3}{|l|}{ Ramachandran plot } \\
\hline Favored (\%) & 97.0 & 97.4 \\
\hline Allowed (\%) & 3.0 & 2.6 \\
\hline Outliers (\%) & 0 & 0 \\
\hline Rotamer outliers (\%) & 0.63 & 2.56 \\
\hline Average B-factor $\left(\AA^{2}\right)$ & 48.60 & 64.22 \\
\hline Macromolecules & 48.57 & 64.36 \\
\hline Ligand & $\mathrm{N} / \mathrm{A}$ & 60.98 \\
\hline Solvent & 49.28 & 57.56 \\
\hline
\end{tabular}

A3Fctd9x Zinc-bound

Statistics for the highest-resolution shell are shown in parentheses. 


\section{FIGURES}

\section{Figure1}

(a)

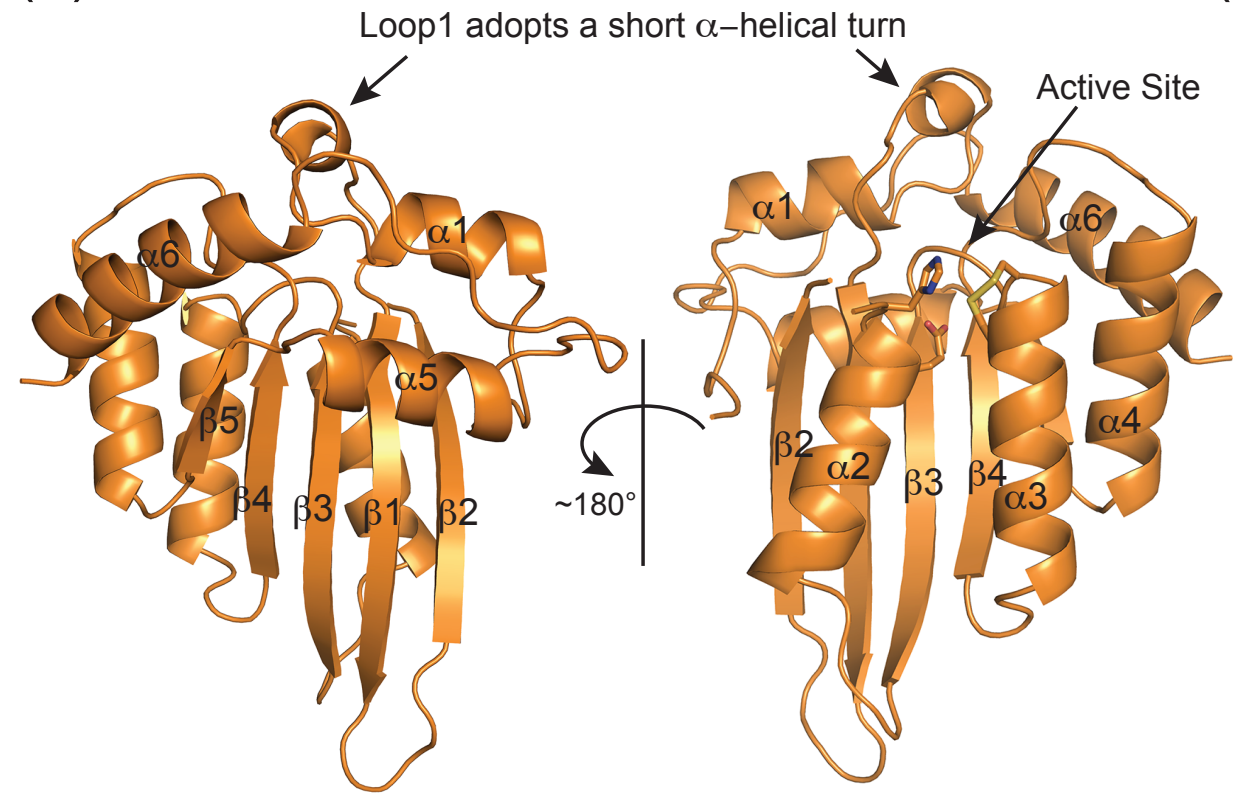

(b)

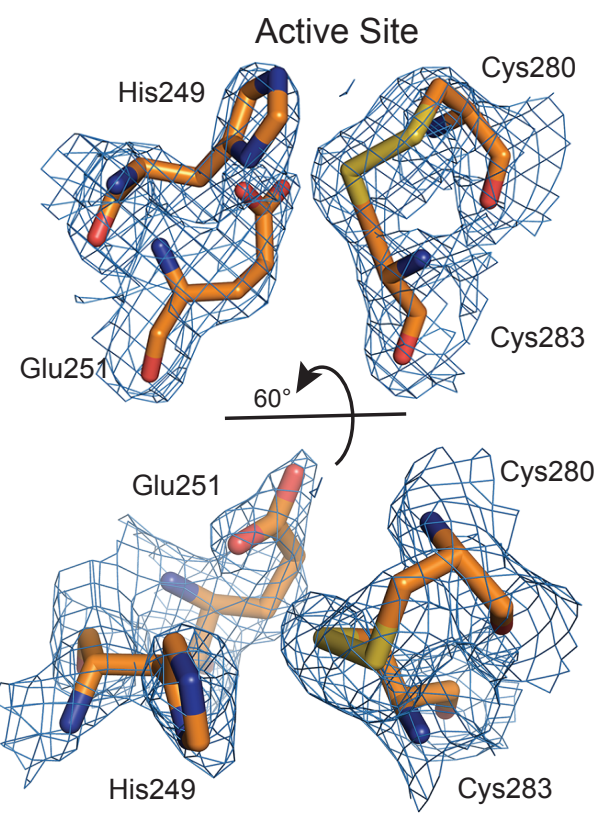

(c)

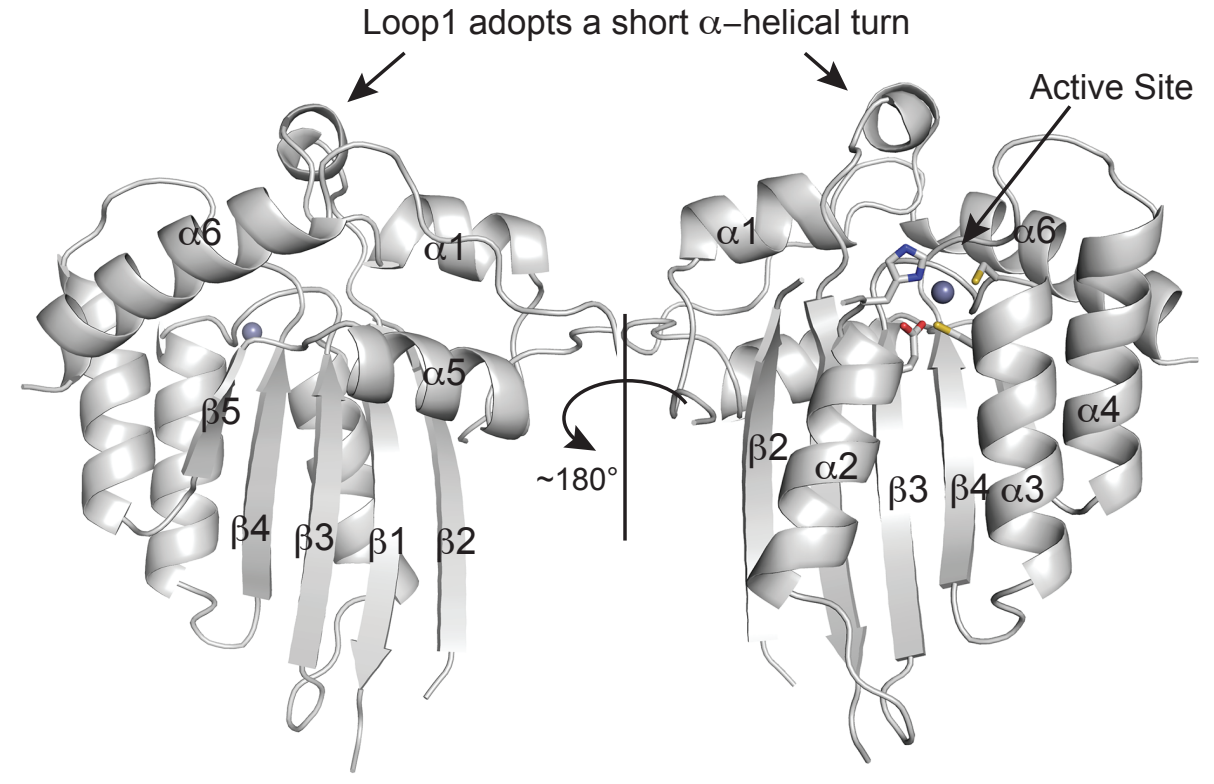

(d)
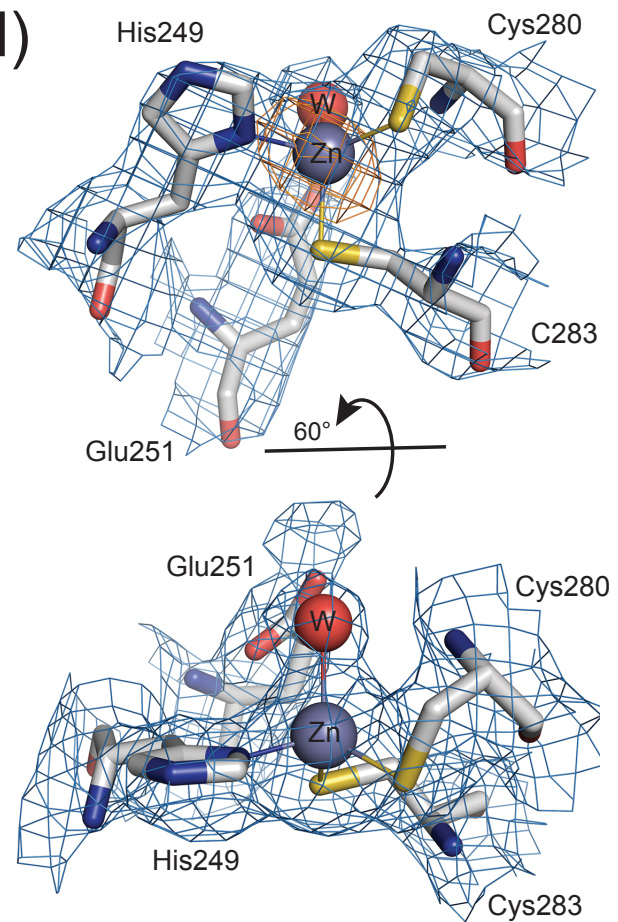
(a)

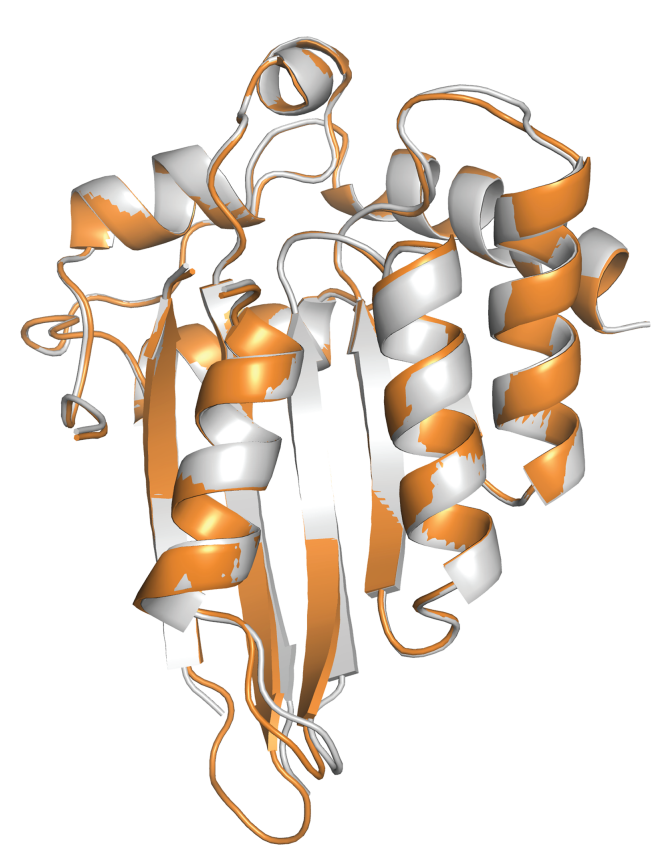

(b)
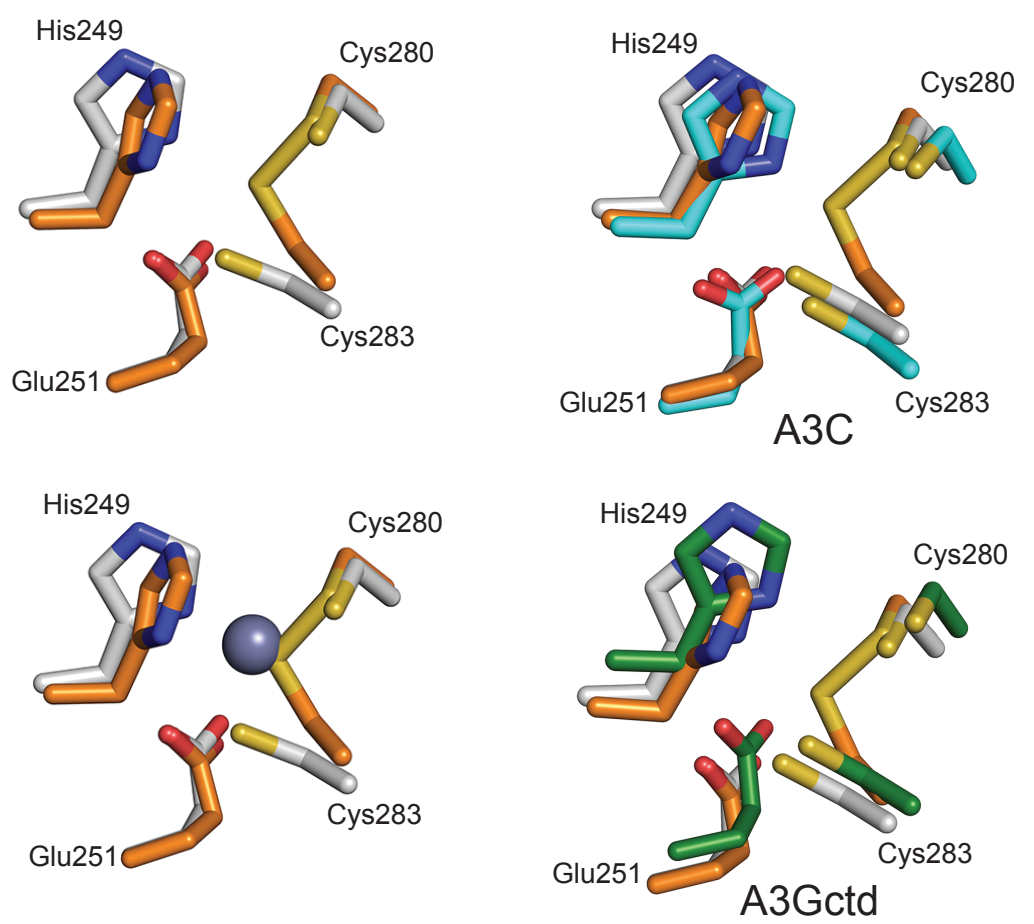
Shaban et. al., Fig. 3

(a)

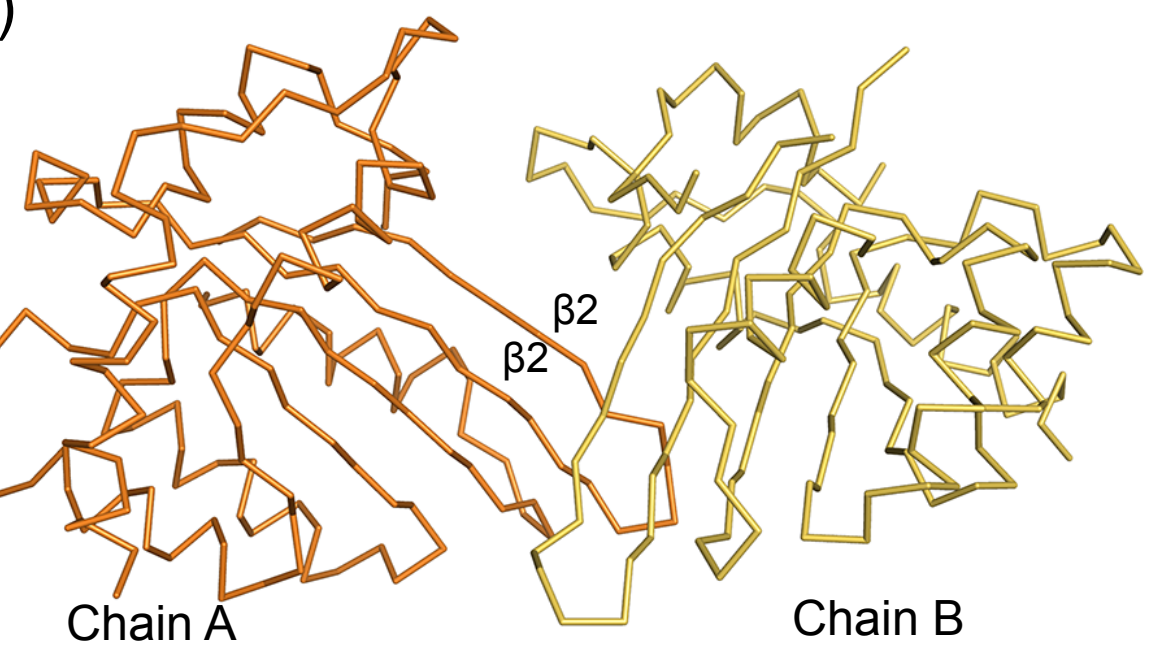

(b)

(c)

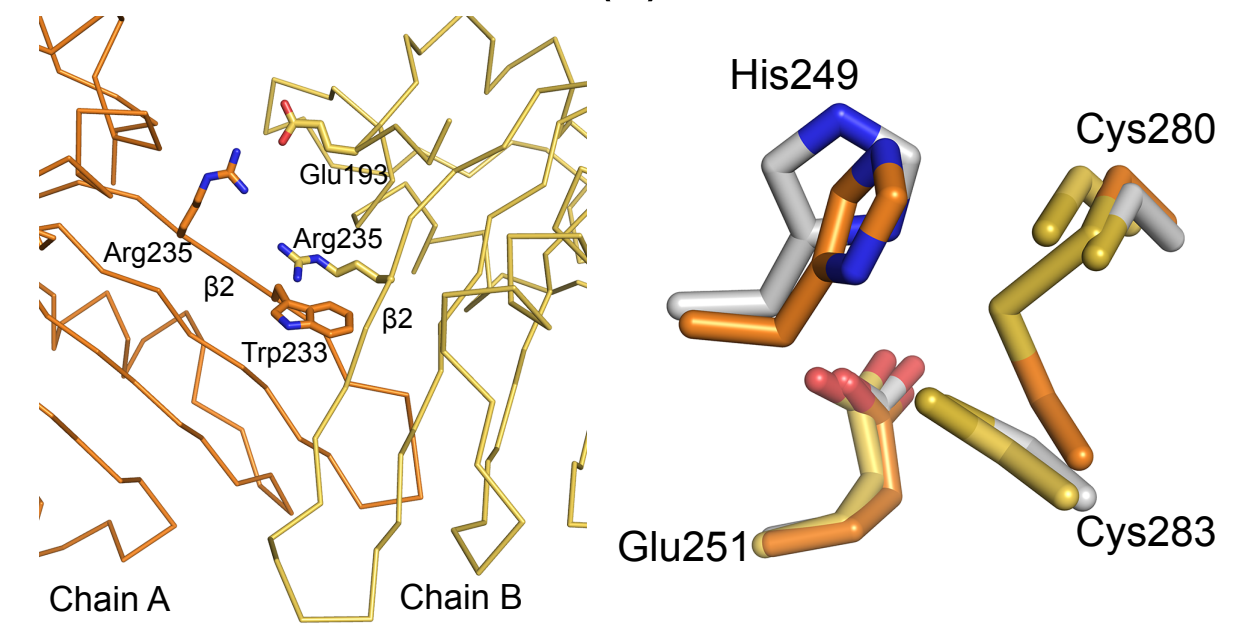


(a)

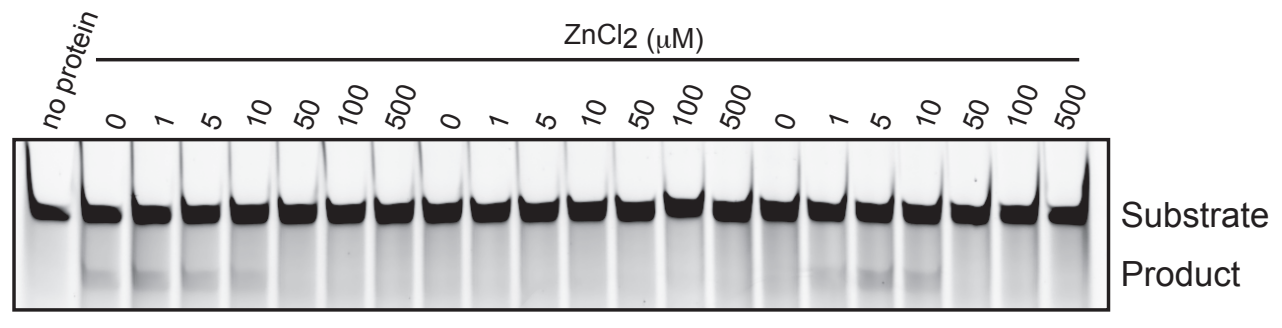

(b)

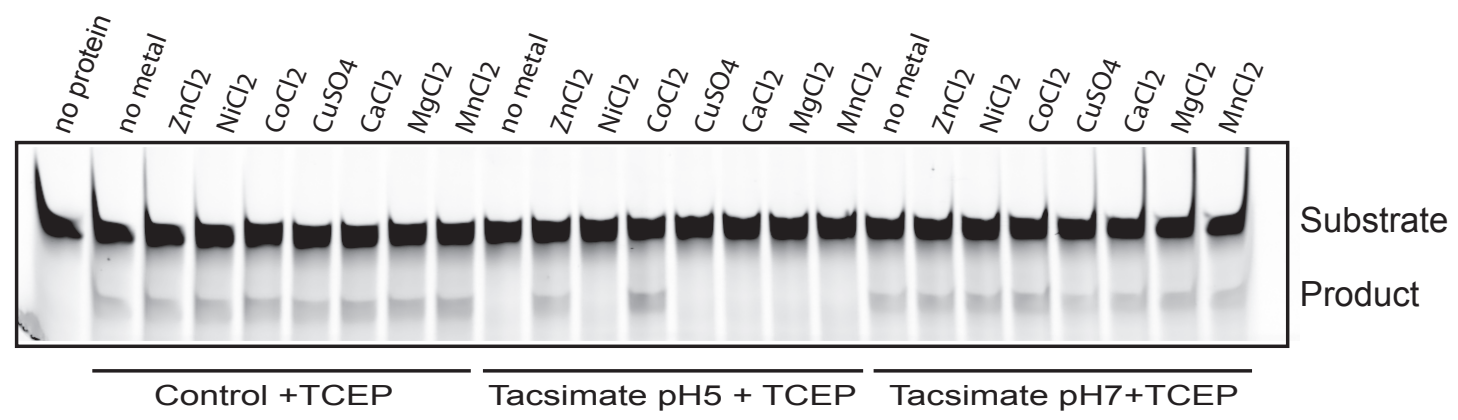

$$
\begin{gathered}
\text { Tacsimate } \mathrm{pH} 5 \quad \begin{array}{c}
\text { Tacsimate } \mathrm{pH} 5 \\
\text { +TCEP }
\end{array}
\end{gathered}
$$

$$
\text { Control +TCEP Tacsimate pH5 + TCEP Tacsimate pH7+TCEP }
$$


Shabanetal_Graphical Abstract

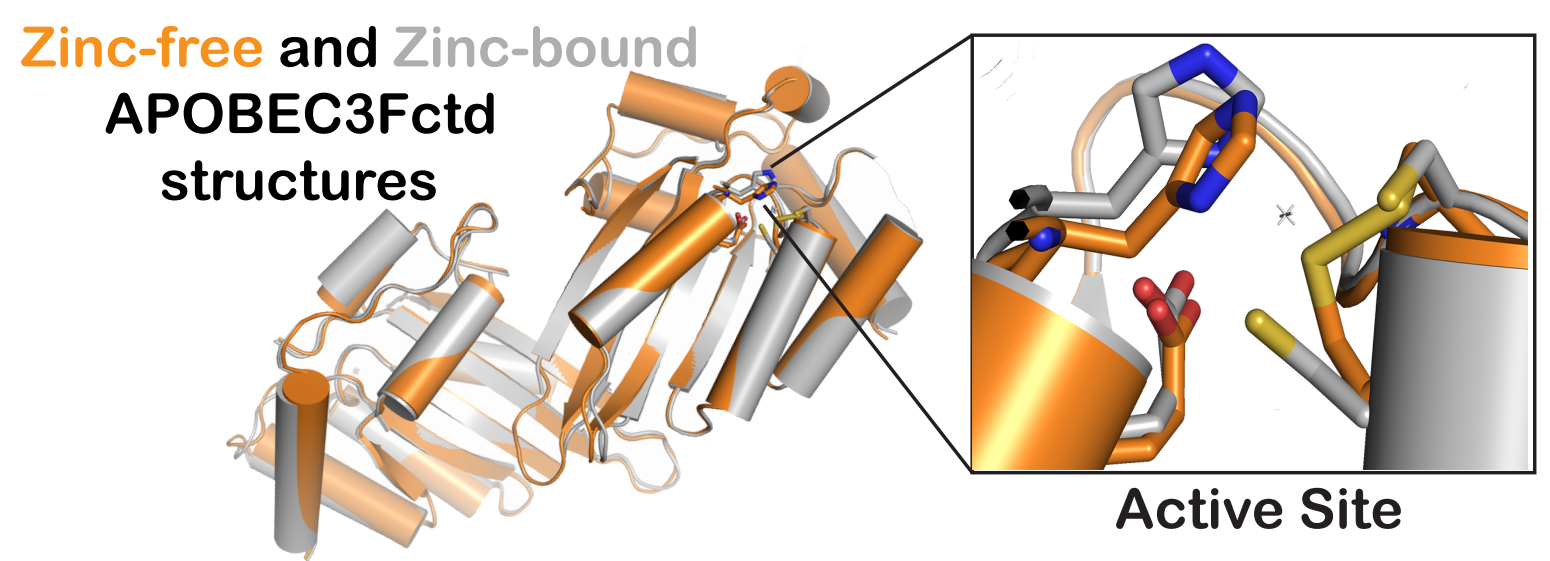

